\title{
An Update on the CCSDS Optical Communications Working Group
}

\author{
Bernard L. Edwards \\ NASA Goddard Space Flight Center \\ Greenbelt, MD 20771 USA \\ Klaus-Juergen Schulz \\ European Space Agency \\ Darmstadt, Germany \\ Jon Hamkins \\ NASA Jet Propulsion Laboratory \\ Pasadena, CA 91109 USA \\ Bryan Robinson \\ MIT Lincoln Laboratory \\ Lexington, MA 02420 USA \\ Randall Alliss \\ Northrop Grumman Corporation \\ McLean, VA 22102 USA
}

\author{
Robert Daddato \\ European Space Agency \\ Darmstadt, Germany \\ Christopher Schmidt \\ DLR \\ Oberpfaffenhofen, Germany \\ Dirk Giggenbach \\ DLR \\ Oberpfaffenhofen, Germany \\ Lena Braatz \\ Booz Allen Hamilton, Inc. \\ McLean, VA 22102 USA
}

\begin{abstract}
International space agencies around the world are currently developing optical communication systems for Near Earth and Deep Space applications for both robotic and human-rated spacecraft. These applications include both links between spacecraft and links between spacecraft and ground. The Interagency Operation Advisory Group (IOAG) has stated that there is a strong business case for international cross support of spacecraft optical links. It further concluded that in order to enable cross support the links must be standardized. This paper will overview the history and structure of the space communications international standards body, the Consultative Committee for Space Data Systems (CCSDS), that will develop the standards and provide an update on the proceedings of the Optical Communications Working Group within CCSDS. This paper will also describe the set of optical communications standards being developed and outline some of the issues that must be addressed in the next few years. The paper will address, in particular, the ongoing work on application scenarios for deep space to ground called High Photon Efficiency, for LEO to ground called Low Complexity, for inter-satellite and near Earth to ground called High Data Rate, as well as associated atmospheric measurement techniques and link operations concepts.
\end{abstract}

\section{International Interoperability of Radio Frequency Communications}

At the start of the Space Age, each international space agency began developing its own space communication capabilities to communicate with its spacecraft. To achieve global coverage for its spacecraft, each agency had to build ground stations around the Earth. This became a very expensive thing to do that only the larger space agencies could afford; also, it became apparent to all that communications coverage and availability could be increased by sharing communications infrastructure around the world. The key to allowing this "cross support" (see Figure 1) is communications standards; by developing interoperability standards, one space agency's spacecraft could be served by another space agency's ground antennas.

Today, the overall development of international space communication standards for cross support is coordinated by the Interagency Operations Advisory Group (IOAG) [1]. The IOAG is an organization made up of international space agencies that provides a forum for identifying common needs and coordinating space communications policy, high-level procedures, technical interfaces, and other matters related to 


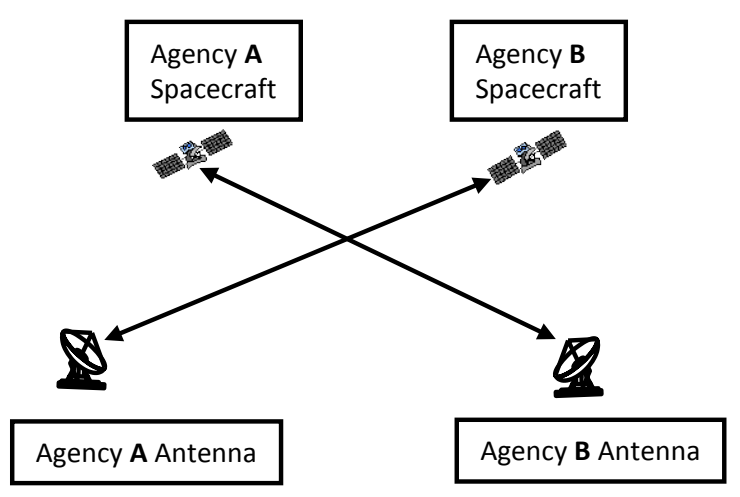

Figure 1: Cross Support

interoperability and space communications. The IOAG meets face to face at least annually and has

several telecons throughout the year. Over the years, it has established effective liaisons with key enabling groups for interoperability, including the Consultative Committee for Space Data Systems (CCSDS), the Space Frequency Coordination Group (SFCG), the International Committee on Global Navigation Satellite Systems (ICG), and the International Space Exploration Coordination Group (ISECG). The IOAG considers the future requirements and trends in spacecraft communications needs and assigns priorities for the development of cross support standards. The standard development is then accomplished by the CCSDS, a multi-national forum comprised of the world's major space agencies and observer agencies. The stated goal of the CCSDS is to enhance governmental and commercial interoperability and cross support, while also reducing risk, development time, and project costs. Consensus must be reached by the member agencies before a CCSDS standard can be published.

Since its founding CCSDS has developed standards recommendations, which have become International Standards Organization (ISO) standards, for space link communications and for associated ground data systems. These standards enable interoperability and cross support among the international space agencies.

As the standards have been developed over the past 30 years, the international space agencies have gradually updated their ground space communication antennas and ground data systems to implement the cross support standards. As more and more systems have adapted the CCSDS standards, the agencies have begun to enjoy the benefits of cross support. Options for spacecraft communications have increased and the cost of supporting a single agency's spacecraft has been reduced, as agencies use the communications assets of other entities to fulfill their spacecraft's communication needs.

\section{The Development of Space}

Communication Standards within CCSDS

The CCSDS member and observer agencies contribute technical experts to develop space data standards. The CCSDS has established a number of Working Groups that are focused on specific topics. Each CCSDS Working Group has a leader, chosen from a member agency, and a group of experts contributed by member agencies interested in the products being produced by that Working Group. The products are documents that recommend standard communication protocols, procedures, and concepts that will enable cross support in the particular area of interest of the Working Group. Often there will be multiple subtopics to be addressed by the Working Group so it may organize subgroups to deal with those specific subtopics. Subtopics subgroups usually produce a document that captures the final results of their studies and deliberations.

The Working Groups meet in face-to-face meetings twice a year. A lot of work is also conducted between those face-to-face meetings via telecons and emails.

The products from the Working Groups are colorcoded according to the following guide:

- Blue Books are completed recommended standards that become ISO standards. They are normative, sufficiently detailed, and pre-tested so they can be used to directly and independently implement interoperable systems.

- Magenta Books describe recommended practices. They are normative, but at a level that is not directly implementable for interoperability. These are reference architectures, application program interfaces, operational practices, etc.

- Green Books are informational reports. These are not normative. These may be foundational for Blue and Magenta Books, describing their applicability, overall architecture, concept of operations, etc.

- Red Books are working copies of the recommended standards before they are promoted to the Blue Book or Magenta Book level. These should be used with caution as they can change before officially released. 
- White Books are the initial conceptual working draft documents in a topic area.

- Orange Books document experimental work. They are normative, but generally cover very new technology that does not yet have consensus of enough member agencies to standardize.

- Yellow Books are administrative books. They document CCSDS procedures, proceedings, test reports, etc.

- Silver Books are historical books. They are retired documents that are kept available to support existing or legacy implementations. The implication is that other agencies may not provide crosssupport.

- Pink Books/Pink Sheets are draft revisions to Blue or Magenta Books that are circulated for agency review. Pink Books are reissues of the full book while Pink Sheets are change pages only.

Once Blue Books are finalized they are brought to an ISO Standards subgroup where they are promoted to ISO Standards. ISO Technical Committee 20 Subcommittee 13 (TC 20/SC 13) is the ISO administrative subcommittee of CCSDS. By special arrangement with ISO, CCSDS documents are processed as ISO TC 20/SC 13 projects at the Draft International Standard (DIS) stage. Effectively, the CCSDS membership now has a dual role, functioning as the CCSDS standards body and as the ISO TC 20 /SC 13 standards body.

\section{Optical Communications}

In recent years, there have been significant advancements in the development of laser-based communication systems for space applications. These optical communication systems hold the promise of better than an order of magnitude higher data rates over radio frequency (RF) space communications, while using less power, having lower mass, and occupying less space than comparable RF communication systems.

In 2010, the IOAG commissioned a study on the business case for establishing cross support standards for optical communications. The study group was named the Optical Link Study Group (OLSG) and it was co-chaired by the National Aeronautics and Space Administration (NASA) and the European Space Agency (ESA).
The OLSG had six member agencies and assessed the business case by defining mission scenarios, developing a credible operational concept for each scenario, and examining the corresponding space communication system designs, estimated costs, and their expected performance. Some of the scenarios that the OLSG looked at were Earth relay satellites, Low Earth Orbit (LEO) direct-to-ground, lunar direct-to-Earth, and deep space optical directto-Earth communications.

Earth relay satellites are satellites placed in Earth orbit to relay signals from other spacecraft, airplanes, balloons, etc. to centralized ground stations. Earth relay satellites are expensive to develop, build, and operate. Sharing of an Earth relay satellite will reduce the cost of providing worldwide coverage for optical inter-satellite communication links. Sharing of Earth relay satellites should also provide higher availability by making more resources available to a specific spacecraft.

While optical ground stations to support Near Earth missions are much less expensive to develop and build, sharing those resources will also help to make optical communications more reliable and affordable. Optical communications through the Earth atmosphere is nearly impossible in the presence of most types of clouds. Therefore, the optical communication system solution for a particular mission has to utilize optical ground stations that are geographically diverse, such that there is a high probability of a cloud-free line of site (CFLOS) to a ground station from a spacecraft at any given point in time (e.g., at the same longitude or at a sufficient number of stations at different longitudes to allow the stored onboard data to be transmitted within the allocated time). Sharing ground stations around the world helps to increase the probability of getting the data to the ground within the time period required.

Optical ground stations to support deep space direct-to-Earth links are expected to be large, relatively expensive ground stations. This is especially true for stations supporting very high data rates from Mars and beyond. The effective aperture size will be on the order of 5 to 10 meters to support high rate optical signals from Mars and even larger for signals from farther out in the solar system. Just as in the Near Earth application case, the sharing of deep space optical ground stations will save cost and increase availability. This will help to make future deep space optical communications a reality, as the sharing of infrastructure will lower the cost for any single space agency. 
In all of the scenarios examined, the OLSG found that "cross support will allow sharing of the cost and usage of the global optical terminal infrastructure needed to serve future missions, and will boost missions' scientific return.” [2] The OLSG completed its Final Report in 2012, with an official addendum with more details added in 2013, stating that a business case had been found for optical communication cross support. The OLSG recommended actions to be taken to advance the development of interoperable international standards for cross support, including the creation of a CCSDS Working Group focused on optical communications.

\section{The CCSDS Optical Communications Working Group}

As requested by the IOAG, the CCSDS officially formed an Optical Communications Working Group to develop world-wide standards for space optical communications. The working group is chaired by NASA with a deputy from ESA and it had its kick-off meeting in January 2014. The working group plans to develop:

- New standards in wavelength, modulation, coding, interleaving, synchronization, and acquisition, which are likely different from existing RF standards.

- New standards for definition, exchange, and archiving of weather data for predicting and operating optical communication links among optical ground stations and their network operations centers.

Standards specifically for space optical communications are required for the modulation, coding, interleaving, synchronization, and acquisition of signals and will have to take into account the severe impact of the Earth's atmosphere on space-to-ground links. The atmospheric impacts on the link are typically more severe than the corresponding impacts on RF links. Several space agencies are developing optical communications terminals that can support both space-to-ground and space-to-space links and the objective is to develop maximum synergy, as far as practical, between the various scenarios.

The working group has been examining three different scenarios for optical communications: High Data Rate, High Photon Efficiency, and Low Complexity. High Data Rate links are expected to be used for Near Earth applications where extremely high performance is desired. High Photon Efficiency links are expected to be used for deep space direct-to-Earth links and are basically "photon starved" links; these links could also be used in Near Earth applications where there is limited mass and power to support communications, such as on a CubeSat. It is expected that the underlying technologies and techniques for modulation, coding, and synchronization will be significantly different between the two signal cases. Finally, the working group is discussing a possible recommendation for Low Complexity systems, where perhaps cost or simplicity is more important that high performance, efficiency, or data rate.

In addition to the typical standards that have to be developed for any communications system, such as modulation and coding, space optical communications also require a standard for the definition, exchange, and archiving of weather and atmospheric data. That is because optical space communications through Earth's atmosphere are adversely affected by the presence of cloud cover and optical turbulence, and to a lesser extent, by aerosols in the atmosphere. These atmospheric challenges to operational optical communications systems are illustrated in Figure 2, which shows geostationary (GEO) and low Earth orbiting (LEO) satellites interacting with and working around atmospheric degradations. GEO and LEO satellites are shown orbiting the Earth. Optical links are shown by the cones: dark green represents a Cloud Free Line of Sight (CFLOS) and thus full data rate link, light green represents a link degraded by turbulence, orange represents a link attenuated by aerosols, red represents a link blocked by clouds, and yellow indicates an uplink from a LEO to a GEO.

Each of these atmospheric effects may require one or more different mitigation strategies, which may, in turn, be dependent on the operational design of a particular system.

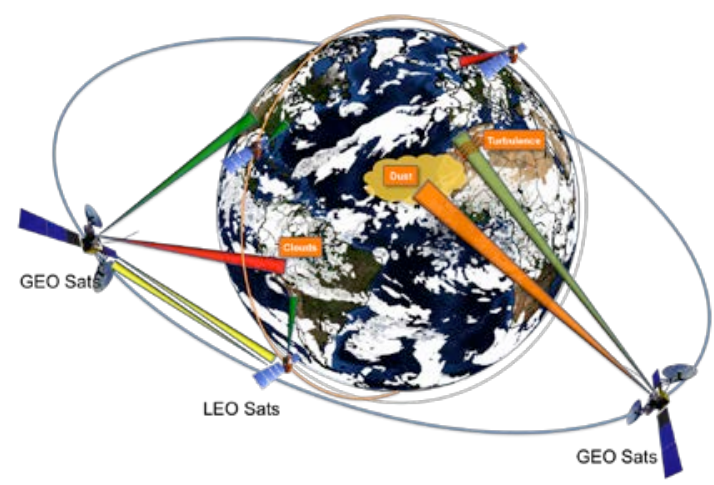

Figure 2: Conceptual Illustration of Atmospheric Effects experience by space-toground optical communications 
Because space-to-ground optical communications are affected by the presence of cloud cover and other atmospheric effects it is important to specify, accurately measure, analyze, characterize, and ultimately predict critical atmospheric parameters for the purposes of: selection of Earth Terminal Sites (ETS), development of concepts of operation-including real-time knowledge of atmospheric parameters needed to make link handover decisions, evaluation of the long-term atmospheric characteristics of ETS, and development of link budgets.

One objective of the CCSDS optical communication working group is to develop standards for the development of local instruments that can characterize the atmospheric channel, as well as techniques on how to use the data to optimize and inform link handover decisions.

The working group is developing the following CCSDS books:

1) Blue Book on Optical Communications Physical Layer

2) Blue Book on Optical Communications Coding and Synchronization

3) Green Book on Atmospheric Characterization for Optical Communication Systems

4) Magenta Book on Atmospheric Characterization and Forecasting for Optical Link Operations

5) Orange Book on Optical High Data Rate Communications - 1550nm

6) Orange Book on Optical High Data Rate Communications - 1064nm

\section{The Blue Books}

The Blue Book on Optical Communications Physical Layer will define the physical layer parameters and techniques required for interoperability of optical communications. The Blue Book on Optical Communications Coding and Synchronization shall define the coding, synchronization, interleaving parameters and techniques required for interoperability of optical communications. The first version of both books will cover the High Photon Efficiency (HPE) scenario; subsequent versions will cover the Low Complexity scenario. The German Aerospace Agency, DLR, has volunteered to be the CCSDS Book Editor for the Physical Layer book while NASA has volunteered to be the book editor for the Coding and Synchronization book.

\section{A. High Photon Efficiency}

High Photon Efficiency optical communications are needed for applications in which power efficiency is the dominant consideration in link design. Figure 3 shows a representative Deep Space optical communications architecture requiring high photon efficiency. The deep space optical communications architecture shown in Figure 3 is a primary application expected to require high photon efficiency. Desired features of the HPE specification include a near-capacity channel coding scheme, modularity between Open Systems Interconnection (OSI) layers, flexible parameters that enable HPE operation at a variety of signal and background fluxes, robustness to atmospheric fading, and support for a wide range of data rates.

The CCSDS Optical Communications Working Group has completed a preliminary specification for HPE optical communications. On the downlink, the specification uses a pulse-position modulation (PPM) scheme intended to be received with a direct-detection receiver. The specification includes a serially-concatenated convolutionally-coded PPM (SCPPM) channel code [3], along with an appropriate interface to the data link layer above it. A channel interleaver is used to combat atmospheric fading, and guard slots are used for compatibility with existing laser technology and to aid in synchronization. The system supports data rates between $16 \mathrm{kbps}$ and $2.1 \mathrm{Gbps}$.

The SCPPM code operates within about $1 \mathrm{db}$ of capacity, as shown in Figure 4. The allowable PPM orders, code rates, slot widths, and symbol repeat factors are shown in Table 1.

On the uplink, the specification includes a beacon intended to be tracked by the spacecraft as part of its pointing system. Optionally, a low-rate data rate signal is supported, using a 2-PPM signal encoded with a low-density parity-check code and an optional channel interleaver.

At the physical layer, downlink transmission occurs at $1530-1577 \mathrm{~nm}$, and uplink transmission at 1064, 1070, or $1030 \mathrm{~nm}$. Appropriate center frequency tolerance, laser line width, in-band and spillover emissions, polarization, extinction ratio, timing jitter, pulse shape, and pulse repetition rates are described in the draft specification. 


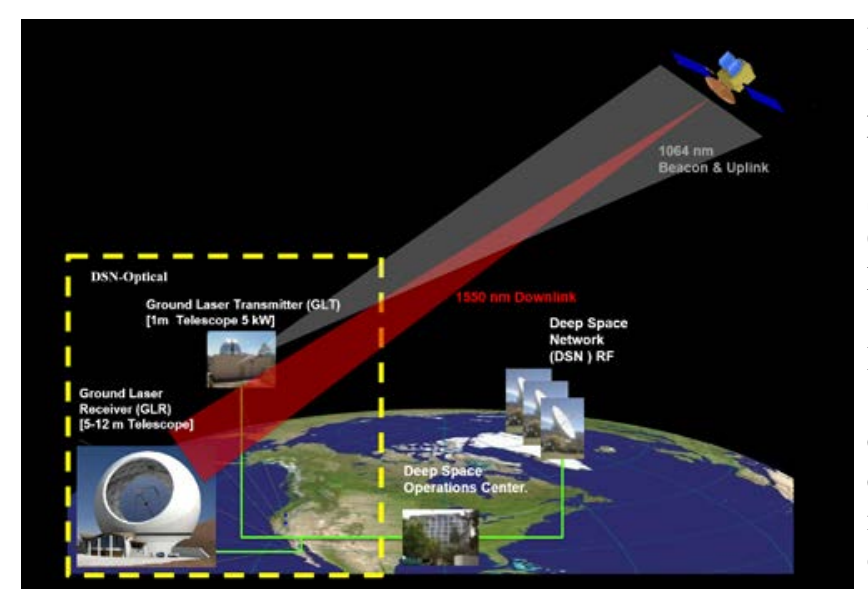

Figure 3: Representative HPE optical communications architecture

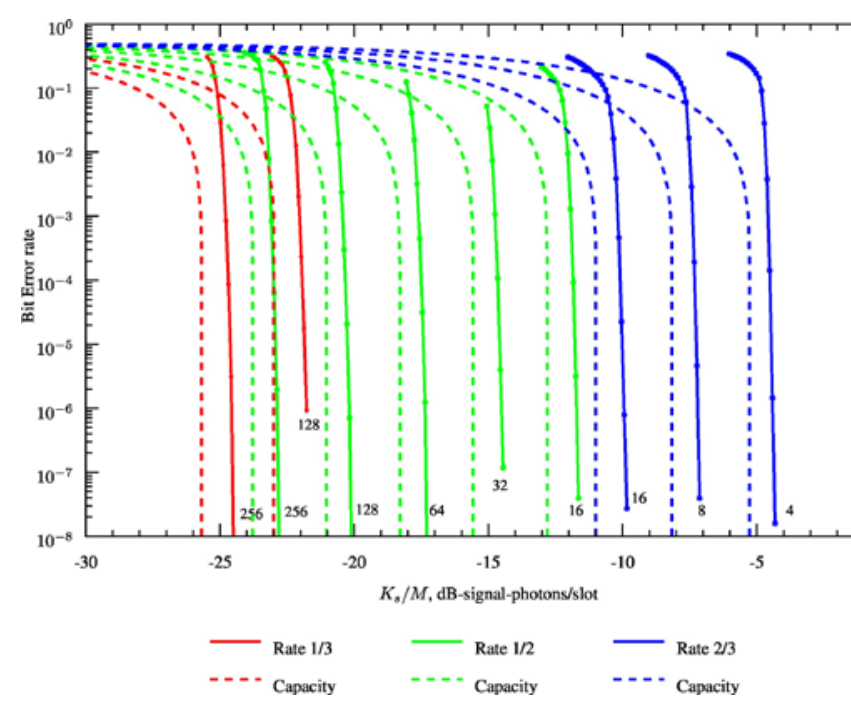

Figure 4: SCPPM performance, for $\mathrm{Kb}=0.01$

\begin{tabular}{|l|l|}
\hline PPM order & $\begin{array}{l}4,8,16,32,64,128, \\
256\end{array}$ \\
\hline Code rate & $1 / 3,1 / 2,2 / 3$ \\
\hline Slot width (ns) & $\begin{array}{l}1 / 8,1 / 4,1 / 2,1,2,4,8, \\
512\end{array}$ \\
\hline Repeat factor & $1,2,4,8,16,32$ \\
\hline
\end{tabular}

Table 1: Allowable parameters values in HPE downlink specification

\section{B. Low Complexity}

The CCSDS Optical Communications Working Group members intend for subsequent versions of the Blue Books to cover what the Working Group currently refers to as the "low complexity" scenario. The low complexity scenario addresses LEO direct-to-Earth (DTE) optical communication links where the reduction of complexity, costs, size, weight, and power takes priority over performance maximization.

Several agencies participating in the Optical Communications Working Group-including NASA, ESA, DLR, the French Aerospace Agency (CNES), and the Japanese National Institute of Information and Communications Technology (NICT) - are working to address the optical communications needs of missions in the low complexity scenario and ensure future interoperability of the space and ground segments of such missions.

In this scenario, reduced complexity is achieved by making the best use of specific LEO-DTE scenario boundary conditions such as short ranges below $2000 \mathrm{~km}$ at low elevations, which lead to a moderate range-loss in the link budget. This sqenario allows for a strongly asymmetric link la fayout employing a very compact space terminal in combination with larger antennas at the Optical Ground Station (OGS). Communication is unidirectional-from the satellite terminal to the GGS only-enabling further reduction of complexity in the space terminal by eliminating the need for a data receiver and protocols in the bajckground. The data throughput of the low complexity systems is limited to the gigabit/second (Gbps) range using a simple direct-modulated laser source in the space terminal and a robust intensitymodulated direct detection signal on the ground. Use of larger OGS aperture diameters eliminates the need to perform complex atmospheric corrections on the ground segment.

Low complexity optical DTE links from LEO spacecraft have been demonstrated by JAXA's Optical Inter-orbit Communications Engineering Test Satellite (OICETS) mission and Kirari Optical Downlinks to Oberpfaffenhofen (KIODO) Experiments in 2006 and 2009 [4], and have been continued with the NICT Small Optical TrAnsponder (SOTA) payload onboard the Space Optical Communications Research Advanced Technology Satellite (SOCRATES) micro-satellite in May 2014 [5]. DLR's Optical Space Infrared Downlink System (OSIRIS) is flying on two Earth observation missions, the Flying Laptop Satellite as well as DLR's Bi-spectral InfraRed Optical System (BiROS) satellite [6]. During these missions, optical technology has been verified in parallel to scientific measurements, enabling characterization of the optical downlink channel. Based on this research, coding and error correction can be optimized for application on LEO-DTE links. Therefore, these technology demonstrations have been selected as the basis for the low complexity standard. 
The low complexity standard shall cover a wide range of applications, from the smallest system on a CubeSat, to larger payloads in the $100 \mathrm{~kg}$-satellite class. To ensure the space segments of different vendors are compatible with optical ground stations or ground station networks of different providers, key technical parameters need to be standardized with enough flexibility to ensure robust and innovative implementations. The physical layer book will describe technical parameters like the wavelength and signal definition in the downlink from the space terminal to ground, as well as for an optional beacon system from ground to space. The different concepts that have been implemented will be represented in the standard. In addition, the maximum data throughput will be represented in the maximum channel symbol rate. The synchronization and coding book content will focus on aspects like the modulation format, implementation of error correction techniques according to the requirements set by the optical communication channel, and the reuse of existing techniques from other CCSDS standards.

\section{The Atmospheric Characterization Books}

The Green Book on Atmospheric Characterization for Optical Communication Systems provides a comprehensive description of the critical atmospheric parameters, and describes suggested types of instruments that can be used to measure these parameters. The book includes material showing how to produce and use long-term weather and atmospheric statistics and how to take real-time measurements. The book describes various types of whole sky imagers some of which operate in the visible wavelengths and others in the longwave infrared. In addition, the book describes a ceilometer for the use of atmospheric attenuation measurements, as well as a Differential Imaging Motion Monitor (DIMM) for atmospheric seeing/turbulence measurements. The Japanese National Institute of Information and Communications Technology (NICT) has volunteered to be the CCSDS Book Editor for this book.

The Magenta Book on Atmospheric Characterization and Forecasting for Optical Link Operations will discuss best practices and touch on performing predictive weather in support of optical communications handovers. The scope of this book includes the use of atmospheric prediction techniques on time frames from minutes (LEO scenarios) to hours (Deep Space scenarios). NICT has volunteered to be the CCSDS Book Editor for this book.

\section{The Experimental Books on Optical High Data Rate Communications}

Members of the CCSDS Optical Communications Working Group are working on two CCSDS Orange Books for High Data Rate optical communications. Such links are expected to be used for Near Earth applications where extremely high performance is desired. When the working group was formed, there was a desire to include the High Data Rate scenario in the Blue Book on Optical Communications Physical Layer and in the Blue Book on Optical Communications Synchronization. However, it was impossible to reach consensus on a single recommendation for High Data Rate optical communications; after protracted discussions, it was decided to produce two CCSDS Orange Books.

The Orange Book on Optical High Data Rate Communications - $1550 \mathrm{~nm}$ is being developed by the French Aerospace Agency (CNES), the Japanese Exploration Agency (JAXA), NICT, and NASA. It will focus on future high performance, high data rate, optical communications at $1550 \mathrm{~nm}$.

The Orange Book on Optical High Data Rate Communications - $1064 \mathrm{~nm}$ is being developed by ESA and the German Aerospace Agency (DLR). It will focus on high data rate optical communications at $1064 \mathrm{~nm}$, based on their experience with the European Data Relay System (EDRS).

\section{A. CNES/JAXA/NASA/NICT Orange Book}

The CCSDS Orange Book for High Data Rate Optical Communications - $1550 \mathrm{~nm}$, is being developed by CNES, JAXA, NASA, and NICT. This specification aims to address a variety of nearEarth optical communications link applications, including relay, crosslink, and direct-to-Earth links from ground, airborne, and space users, as depicted in Figure 5, using fiber telecommunications compatible wavelengths in the 1550-nm regime. To support such a wide array of applications, the optical signaling must be very flexible. For relay applications, data transfer from users in low- to medium-Earth orbit to a ground terminal via a relay satellite (or multiple satellites) in geosynchronous orbit is envisioned. Today, such links are designed to operate at hundreds of Mbps to a few Gbps. The Orange Book specification will support these links as well as future relay links at rates of tens to hundreds of Gbps. For direct-to-Earth links, the specification aims to support the higher link rates afforded by short link distances from, say, low Earth orbit to ground. Such links are expected to operate at rates $>100$ Gbps to support large data transfers with short contact times. 
The Orange Book specification being developed leverages design features and lessons learned from ongoing development programs including NASA's Laser Communication Relay Demonstration (LCRD) [7] and JAXA's Japanese Data Relay System (JDRS) [8]. However, the Orange Book specification targets future operational systems and is not necessarily constrained by the design details of those pathfinder systems. High-level features of the Orange Book specification include: CCSDS transfer frames at the data link interface, use of multiple high-efficiency modern forward error correction codes (e.g. DVB-S2, G.975, etc.), framing that supports multi-hop edge-to-edge forward error correction, and variable-rate burstmode phase-shift keying modulation on a $1550-\mathrm{nm}$ carrier, which can be demodulated with either a non-coherent (e.g., differential phase shift keying [DPSK]) or a coherent receiver (e.g., homodyne binary phase shift keying [BPSK]). Data rate flexibility required for the various link applications is provided by varying the code and modulation rates, as well as using standard fiber telecommunications approaches such as Wavelength Division Multiplexing (WDM).

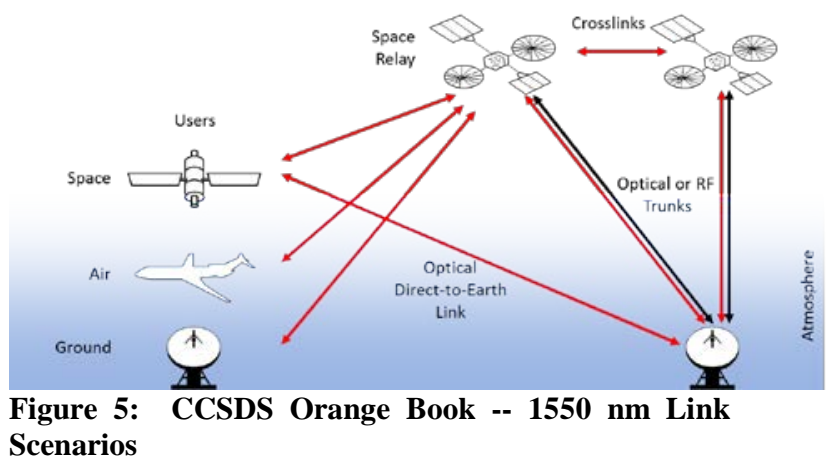

\section{B. ESA/DLR Orange Book}

The purpose of the ESA/DLR Orange Book for Optical High Data Rate (HDR) Communication $1064 \mathrm{~nm}$ is to provide recommendations for optical communication solutions, taking as reference the implementation in current European Space Agency missions (EDRS series, Sentinel series) and the original German Aerospace Agency (DLR) developments.

The key technical features are the use of the 1064 $\mathrm{nm}$ wavelength with BPSK modulation, providing a user data rate of $1.8 \mathrm{Gbps}$.

The Orange Book contains the technical specification for implementing the Physical Layer and the Coding and Synchronization sublayer of optical communications systems supporting the High Data Rate scenario. Among the characteristics that must be implemented to ensure terminal compatibility and successful link acquisition and data transfer are:

- Optical signal: band plan, laser center frequency, tuning range, tuning rate, line width, intensity/frequency/phase noise, polarization, extinction ratio, and modulation scheme; and

- Coding and synchronization: frame structure, channel coding/interleaving/ scrambling, frame synchronization, multiplexing.

Future evolution of the Orange Book is foreseen in the direction of dual-wavelength terminals, increased data rates (3.6-7.2 Gbps), quadrature phase shift keying (QPSK) modulation, and novel coding schemes (to be specified in the context of the ESA ScyLight program, a dedicated new ESA framework on optical communication technology, and the ESA GlobeNet project). In the longer-term future, further scaling of the user data rate can be achieved by implementing WDM to utilize the available Ytterbium amplifier bandwidth of more than $60 \mathrm{~nm}$.

ESA and DLR consider this Orange Book to be a candidate for a future CCSDS recommendation for optical systems interoperable with EDRS, or complementary missions like ESA GlobeNet, or even for other dedicated scenarios.

The described optical communications mechanisms will be useful in particular for LEO-GEO and GEO-GEO Inter-Satellite Link (ISL) operational scenarios. However, they can also be used for space-ground and airborne-space links, if additional measures are taken, e.g., adaptive optics, interleaving, erasure coding.

This Orange Book provides additional proven options for implementation of optical communication for the case of high data rate transmissions, and it should be adopted by ESA for the above-mentioned missions and beyond. Furthermore, the Orange Book will complement the optical communication recommendations for High Photon Efficiency (HPE) and Low Complexity (LC), currently under development at CCSDS, without overlapping them, but adding a sound candidate for future standardization.

\section{Conclusion}

After years of research and demonstrations, space optical communications are ready to support mission critical communications. This technology has the potential to enable new science and exploration missions throughout the solar system. Optical communications can provide increasingly 
higher data rates over comparable RF systems. While the capacity of current and near-term RF communications technology is still increasing, it will be eventually limited by bandwidth allocation restrictions, power requirements, flight terminal antenna size, and weight limitations. A future space communications network should offer both RF and optical communication services. RF can be reserved for those cases where high availability and thus low latency are absolutely required, since optical communications through the atmosphere for space-to-Earth links will always be impacted by clouds. For space-to-Earth links, optical communications can be reserved for scenarios in which a potential delay in reception is not a problem; in space-to-space links, optical communications can provide both high data rates and high availability.

As international space agencies invest in optical communications there will be an increasing need for cross support between the agencies. Collaboration in optical communications will lower mission cost and risk and likely enable missions which otherwise are unaffordable by one nation on their own. A comprehensive set of standards for space optical communications needs to be developed to enable inoperability. This is the evolutionary next step to today's RF cross support.

\section{Acknowledgments}

Within NASA, optical communications physical coding, and synchronization standards development is being done at NASA's Goddard Space Flight Center, at the Massachusetts Institute of Technology's Lincoln Laboratory in Lexington, Massachusetts, and at the California Institute of Technology's Jet Propulsion Laboratory in Pasadena, California, under contract with NASA. Atmospheric work is being done by Northrop Grumman Corporation under contract to NASA. NASA's work is funded by the Space Communications and Navigation Program Office within NASA's Human Exploration and Operations Mission Directorate.

The low complexity scenario work has been led by DLR's Institute of Communications and Navigation (IKN) in Oberpfaffenhofen, Germany.

ESA is accomplishing optical communications work at facilities in Darmstadt, Germany and Noordwijk, in the Netherlands, and at its optical ground station in Tenerife in the Canary Islands.

\section{References}

1. Jean-Marc Soula, et al. "The Interagency Operations Advisory Group (IOAG) - A decade of leadership in International Space Cooperation,” presented at the SpaceOps 2012. A complete list of authors include Jean-Marc Soula/CNES ; Philip Liebrecht/NASA; Martin Pilgram/DLR ; Jon Walker/NASA; James Costrell/NASA (Retired) ; Gian-Paolo Calzolari/ESA ; Wolfgang Hell/ESA.

2. Interagency Operations Advisory Group, "Optical Link Study Group Final Report," IOAG.T.OLSG.2012. 5 June 2012.

3. B. Moision and J. Hamkins, "Coded Modulation for the Deep-Space Optical Channel: Serially Concatenated Pulse-Position Modulation,” IPN Progress Report 42-161 May 15, 2005.

4. T. Jono, Y. Takayama, N. Perlot, D. Giggenbach, et al, "Report on DLR-JAXA Joint Experiment: The Kirari Optical Downlink to Oberpfaffenhofen (KIODO)," JAXA and DLR, ISSN 1349-1121, 2007.

5. A. Carrasco-Casado, H. Takenaka, D. Kolev, et al, "LEO-to-ground optical communications using SOTA (Small Optical TrAnsponder) Payload verification results and experiments on space quantum communications," Acta Astronautica Vol. 139, 2017.

6. C. Schmidt, M. Brechtelsbauer, F. Rein, C. Fuchs, "OSIRIS Payload for DLR's BiROS Satellite," International Conference on Space Optical Systems and Applications - ICSOS, Kobe, Japan, 2014.

7. B. Edwards, et al., "Overview of the Laser Communications Relay Demonstration Project,” Proc. SpaceOps, 2012.

8. Y. Chishiki, "Overview of Optical Data Relay System in JAXA,” Proc. SPIE vol. 9739, 2016.

9. J. Rush and K. Schulz, "Results of the Optical Link Study Group,” Space Operations 2012 Conference, 2012.

10. R. Alliss and B. Felton, "The mitigation of Cloud Impacts on Free Space Optical Communications,” SPIE Proceedings, Vol 8380, 83819, 2012.

11. A. Biswas, B, Oaida, K. Andrews, et al, “Optical Payload for Lasercomm Science (OPALS) Link Validation During Operations from the ISS,” SPIE Proceedings Vol. 9354, 2015.

12. D. Giggenbach, A. Shrestha, C. Fuchs, C. Schmidt, F. Moll, "System Aspects of Optical LEO-to-ground Links,” ICSO Proceedings. International Conference on Space Optics, 1821 Oct. 2016. 